\title{
Résistance en traction des agrégats terreux: influence de la texture, de la matière organique et de la teneur en eau
}

\author{
Jérôme GUERIF \\ avec la collaboration technique de Jean ROYÈRE \& Denis GRISON (*) \\ I.N.R.A., Station de Science du Sol, Centre de Recherches d'Avignon, Domaine Saint-Paul, B.P.91, F84140 \\ Montfavet
}

RÉSUMÉ

\begin{abstract}
L'hétérogénéité et la discontinuité des matériaux terreux nécessitent de définir l'échelle à laquelle on détermine leur résistance en traction, si on veut interpréter correctement cette variable et l'utiliser dans une modélisation de comportements mécaniques des couches de surface des sols. Une estimation de la résistance en traction résultant pour une grande part de l'organisation des constituants élémentaires est proposée comme élément de référence. Cette résistance en traction «texturale» est étroitement corrélée à la teneur pondérale en argile. Une interprétation à partir de la porosité lacunaire est proposée.

L'effet d'une modification de la teneur en matières organiques sur la résistance en traction texturale moyenne d'un matériau s'avère de faible intensité. Cependant, pour un échantillon dont le rapport $\mathrm{MO} / \mathrm{A}$ moyen est supérieur à 0.9 , l'étalement de la distribution des résistances en traction des agrégats est étroitement dépendant de leur teneur en matières organiques.

La résistance en traction varie très rapidement avec l'humidité : l'essentiel de la variation se situe entre l'état sec et un potentiel compris entre $5 \cdot 10^{2}$ et $10 \cdot 10^{2} \mathrm{kPa}$ au-delà duquel la méthode n'est plus valide.

Un modèle prédictif de $\mathrm{T}$ est proposé, les variables d'entrée se limitant à la teneur en argile, et à la teneur en eau de la phase argileuse.
\end{abstract}

Mots clés additionnels : Rupture, compression simple, organisation texturale, porosité lacunaire, modèle.

Tensile strength of soil aggregates : influence of clay and organic matter content and variation with water content.

Because of soil heterogeneity and discontinuity, the scale at which tensile strength is determined has to be defined. The tensile strength arising from the packing and cementing of the elementary particles is proposed as a reference. An estimate of this « textural " tensile strength was shown to be correlated with clay content (weight per weight). An interpretation of this result, based on an estimate of the volume of the lacunar pores, is proposed. There was apparently only a slight effect of organic matter content on mean « textural » tensile strength. However, within a sample with an organic matter/clay content ratio greater than 0.9 , the spread of the distribution of tensile strengths of individual aggregates depended on organic matter content. Tensile strength varied very rapidly with the water content, decreasing from the dry state to a water potential of $5-10 \cdot 10^{2} \mathrm{kPa}$. A predictive model is proposed, with clay and water contents as the main variables.

Additional key words : Rupture, uniaxial compression, textural porosity, lacuna pores, model.

\section{INTRODUCTION}

La résistance en traction est considérée, généralement, comme la grandeur la mieux corrélée aux propriétés

*Etudiant à l'I.N.A. Paris-Grignon ; D.A.A. Sciences et Techniques des productions végétales (1985). mécaniques des couches de surface telles que : la résistance des éléments structuraux à la désagrégation par l'eau, la résistance des croûtes de battance à l'émergence des plantules (PANABOKKE \& QUIRK, 1957; QUIRK \& PANABOKKE, 1962 ; RogOWSKI et al., 1968 ; RoGOWSKI \& Don KirkHAM, 1976), la résistance au compactage de massifs d'agrégats (DEXTER, 1975; BRAUNACK et al., 1979) ainsi que la résistance à la rupture, par les pièces 
travaillantes, des éléments structuraux (FARREL et al., 1967 ; GodwIN \& Spoor, 1977).

Le caractère fragmentaire et agrégé de la structure des sols cultivés ne permet pas d'appliquer directement les concepts classiques de la résistance des matériaux continus. Cet aspect discontinu et hétérogène implique, notamment, l'introduction d'un facteur d'échelle. En effet le niveau d'organisation auquel se situe la mesure, détermine sa signification. On se propose dans ce travail d'étudier, à l'échelle de l'assemblage des constituants granulométriques élémentaires, quelques-uns des facteurs de variation de la résistance en traction (GuERIF, 1988), tels que les teneurs en argile et en matiere organique, et l'état d'hydratation.

En ayant ainsi défini une résistance mécanique principalement issue de l'organisation texturale, on pourra alors envisager une modélisation et une prévision des comportements précédemment évoqués, à partir cles caractéristiques granulométriques.

\section{MATÉRIEL ET MÉTHODE}

\section{A. Méthode d'estimation de la résistance en traction}

Rogowski (1968) puis DeXTER (1975) ont proposé une méthode de calcul de la résistance en traction à partir des résultats d'un test d'écrasement d'agrégats terreux. Les fondements mécaniques de la relation proposée, ont été établis entre autres, par STERNBERG \& ROSENTHAL (1952) puis par HIRAMATSU \& OKA (1966) pour une sphère soumise à l'action de charges ponctuelles, diamétralement opposées. La sphère se rompt selon un plan diamétral passant par les points d'application des charges. La résistance en traction ( $T$ en $\mathrm{Pa}$ ) est donnée par l'équation suivante :

$$
\mathrm{T}=\mathrm{k}\left(4 \mathrm{~F} / \pi \mathrm{d}^{2}\right)
$$

où :

- F est la force maximum à la rupture (en Newton),

- $\mathrm{d}$ est le diamètre de la sphère, et en pratique la distance entre les points d'application des charges (en $\mathrm{m})$,

- k est un coefficient d'ajustement (sans dimensiori).

On admet que les agrégats :

- sont continus, isotropes, et homogènes,

- ont un comportement élastique, jusqu'au moment de la rupture.

Dexter, en considérant que le coefficient $k$ est indépendant du type de sol ainsi que de l'état d'hydratation des agrégats, tout au moins dans le domaine hydrique de comportement fragile, réduit l'équation (1) à :

$$
\mathrm{T}=0.576 \mathrm{~F} / \mathrm{d}^{2}
$$

\section{B. Technique de mesure de la résistance à l'écrasement}

Notre objectif étant d'apprécier la résistance en traition résultant essentiellement de l'assemblage des cons- tituants élémentaires du sol, on effectuera le test d'écrasement sur des agrégats naturels de 2 à $3 \mathrm{~mm}$. Cette échelle est définie par analogie avec la méthode de mesure de la masse volumique texturale (FIES \& STENGEL, 1981).

Une méthode de détermination de la résistance en traction, fiable et adaptée à l'utilisation en routine, a été établie pour des agrégats naturels à l'état sec (GUERIF, 1988) :

- Les agrégats sont obtenus par fracture ménagée et tamisage entre 3 et $5 \mathrm{~mm}$, de l'échantillon de sol sec à l'air.

- Ils sont arrondis mécaniquement, par usure et brassage, à l'aide d'un disque rotatif abrasif, jusqu'à l'obtention d'une forme aussi sphérique que possible, le diamètre final étant de 2 à $3 \mathrm{~mm}$.

On mesure successivement au cours du test :

- le diamètre des agrégats, avant rupture, avec une butée micrométrique $(10 \mu \mathrm{m})$; cette butée, entraînée par un moteur électrique à une vitesse de $4,33 \cdot 10^{4} \mathrm{~m} \cdot \mathrm{s} !$, sert de piston ;

- la force maximale à la rupture, avec un capteur de force présentant une étendue de mesure de 0 à 5 daN.

\section{Aspects particuliers des mesures à l'état humide}

Un protocole de contrôle de la teneur en eau des agrégats a été utilisé pour atténuer les erreurs dues à l'évaporation en cours de manipulation. On a effectué les mesures sur des lots d'agrégats, et estimé séparément les résistances en traction et les humidités moyennes.

On a procédé comme suit :

- préparation d'un lot d'agrégats à la teneur en eau visée ;

- estimation de la résistance en traction moyenne d'un lot, à partir des mesures individuelles des résistances à l'écrasement d'environ 60 agrégats. Une dérive dans le temps de l'humidité moyenne d'un lot, due à l'évaporation consécutive au renouvellement de l'atmosphère contenue dans le pèse-filtre de stockage, lors des ouvertures successives du couvercle, a été constatée. On a donc été amené à répartir chaque lot dans 6 pèse-filtre (soit environ 300 agrégats par pèsefiltre pour limiter le dessèchement individuel), de chaque pèse-filtre on a extrait seulement 10 agrégats pour les mesures, de manière à limiter le nombre d'ouvertures du couvercle;

- estimation de la teneur en eau moyenne des agrégats de chaque pèse-filtre, à partir de mesures effectuées, sur aliquote, avant et après manipulation. La dessiccation, au cours de la mesure proprement dite, a été négligée.

L'écart-type des distributions des résistances en traction des agrégats amenés à une même humidité, diminue quand cette humidité augmente (fig. 1). On peut donc, à l'état humide, effectuer, par excès, le nombre de mesures de résistance à l'écrasement, jugé nécessaire pour estimer correctement la résistance en traction à l'état sec. Un travail précédent (GUERIF, 1988) a montré qu'avec soixante agrégats environ, on obtient une estimation de la résistance en traction moyenne, à l'état sec, à 10 p. 100 près avec un degré de confiance de 99 p. 100. 
R. en traction en $100 \mathrm{kPa}$

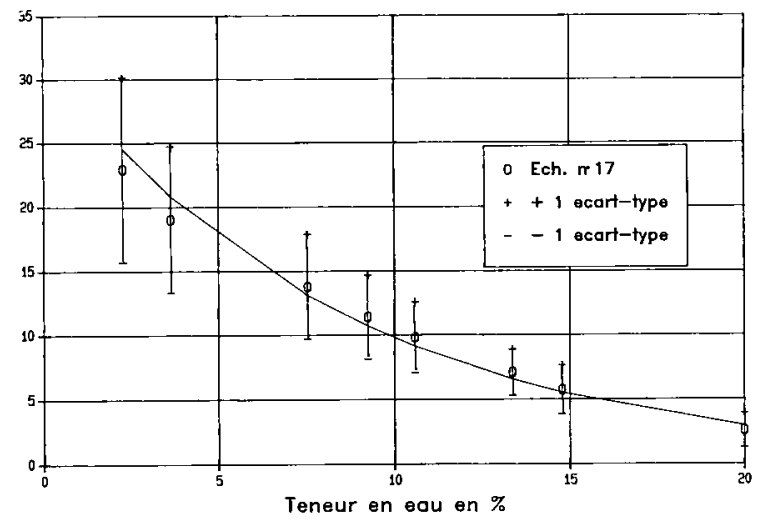

Figure 1

Variation, avec la teneur en eau, de l'écart tye des distributions des résistances en traction des agrégats.

Variation, with water content, of the standard deviation of the distribution of the tensile strengths of the aggregates.

On suit les variations, en fonction du temps, de la force exercée sur l'agrégat avec un enregistreur graphique. L'allure de la courbe est caractéristique du type de comportement à la rupture. Lorsque la courbe présente un pic marqué (fig. 2a) l'agrégat présente un comportement fragile, on admet généralement que l'on satisfait aux conditions d'élasticité, sinon l'agrégat présente un comportement ductile (fig. 2b).

Or pour que l'on puisse estimer une résistance en traction à partir d'une mesure de résistance à l'écrase-
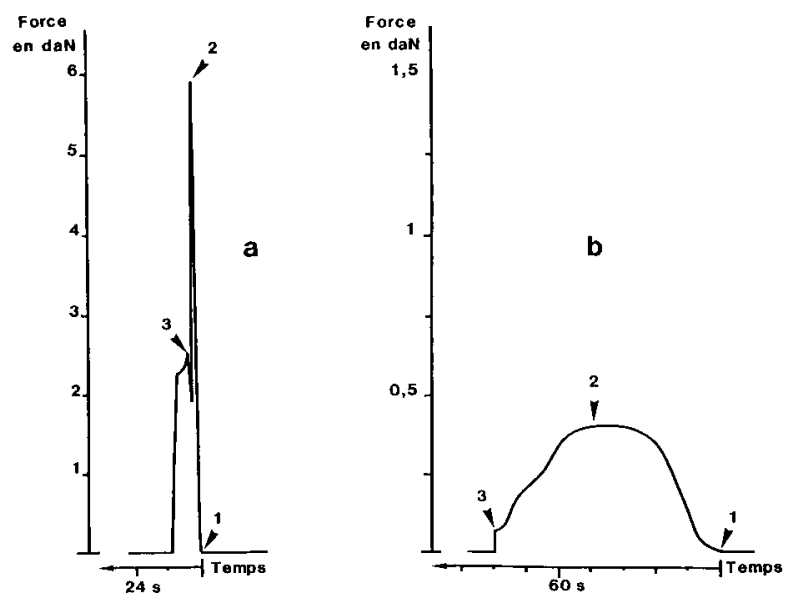

Figure 2

Enregistrement de la force appliquée au cours du test

(1) Début de la mise en charge.

(2) Rupture.

(3) Remise à zéro.

a) Agrégat sec à comportement fragile - validité de l'hypothèsé d'élasticite.

b) Agrégat humide à comportement ductile - non validite de lhypothèse d'elasticile.

Recording the force applied during the test :

(1) Beginning of loading

(2) Rupture.

(3) Unloading

a) Dry aggregate showing brittle rupture - the elastic assumption is valid.

b) Wet aggregate showing ductile rupture - the elastic assumption is no longer valid. ment, il faut que l'hypothèse d'élasticité soit vérifiée et pratiquement :

- il faut qu'une rupture franche soit décelable par le capteur de force, et que l'agrégat présente un plan de rupture net.

- il faut que l'aplatissement de l'agrégat soit suffisamment faible avant rupture, pour que :

- la surface d'application de la charge puisse encore être assimilée à un point, conformément aux hypothèses de calcul.

- l'estimation de la surface de rupture, par la distance entre points d'appui à la mise en charge, ne soit pas biaisée.

A cet égard Frydmann (1964), dans le cadre de l'essai brésilien, a montré que le test reste valide si le rapport entre la largeur de la zone d'aplatissement et la distance entre points d'appui, à la rupture, demeure inférieure à 0,29 . En appliquant ce critère aux agrégats de $2-3 \mathrm{~mm}$, on estime que le diamètre de la zone d'aplatissement ne doit pas excéder $0,6 \mathrm{~mm}$ pour les plus petits, et $0,9 \mathrm{~mm}$ pour les plus grands.

\section{Méthodes de caractérisation des agrégats}

\section{Analyse de l'organisation de l'espace poral textural}

Par analyse de la distribution des diamètres des pores obtenue par porosimétrie à mercure, on peut distinguer (FIES, 1984) 2 sous-espaces poraux :

- l'espace poral lacunaire,

- l'espace poral propre à la phase argileuse.

On peut donc estimer le volume des lacunes, qu'on exprimera en terme de porosité lacunaire soit: le rapport entre le volume de lacune et le volume total.

\section{Maîtrise et contrôle de la teneur en eau des agrégats}

- Après tamisage à sec, les agrégats sont humectés sous vide, sur une plaque de tension, à un potentiel voisin de $0(-0,3 \mathrm{~Pa})$, puis amenés à l'humidité ou au potentiel désiré, soit par dessiccation ménagée sous gel de silice, en enceinte calorifugée, soit par équilibre sur plaque poreuse de RICHARDS.

- Les agrégats « secs» sont préparés sous silicagel, en enceinte calorifugée, jusqu’à poids constant.

- Les teneurs en eau des agrégats sont déterminées par gravimétrie après passage à l'étuve à $105^{\circ} \mathrm{C}$ jusqu'à poids constant.

\section{E. Matériaux}

Les sols choisis (tabl. 1) correspondent au souci d'avoir une gamme de teneurs en argile suffisamment étendue (de 5 à 52 p. 100 d'argile), mais aussi des teneurs en matières organiques suffisamment étalées (le rapport $\mathrm{MO} / \mathrm{A}$ varie de 0,02 à 0,40 ).

Deux limitations interviennent dans l'étude des effets propres aux matières organiques :

- la difficulté de recueillir des matériaux naturels qui, pour une même constitution minérale, présentent une gamme suffisamment étendue de teneurs en carbone. 
TABLEAU 1

Caractéristiques de constitution des materiaux étudiés.

Characteristics of the constitution of the studied soils.

\begin{tabular}{crrr}
\hline \hline Echantillons & $\begin{array}{c}\text { Argile } \\
\text { en \% }\end{array}$ & $\begin{array}{c}\text { MO } \\
\text { en \%o }\end{array}$ & MO/A \\
\hline 1 & 5,7 & 24,40 & 0,43 \\
2 & 11,5 & 16,00 & 0,13 \\
3 & 12,9 & 10,60 & 0,08 \\
4 & 13,8 & 37,80 & 0,27 \\
5 & 15,2 & 9,40 & 0,06 \\
6 & 15,5 & 23,00 & 0,15 \\
7 & 16,1 & 22,30 & 0,14 \\
8 & 16,6 & 20,40 & 0,12 \\
9 & 19,6 & 22,20 & 0,11 \\
10 & 24,7 & 26,60 & 0,11 \\
11 & 24,8 & 7,00 & 0,03 \\
12 & 25,6 & 16,60 & 0,06 \\
13 & 26,7 & 38,50 & 0,14 \\
14 & 30,1 & 24,00 & 0,08 \\
15 & 30,3 & 16,00 & 0,05 \\
16 & 51,5 & 56,50 & 0,11 \\
17 & 51,9 & 16,80 & 0,03 \\
& & & \\
\hline \hline
\end{tabular}

- l'existence, dans les populations d'échantillons de sols cultivés, d'une corrélation entre la teneur en carbone et la teneur en argile, cette dernière étant la variable explicative majeure des propriétés physiques et mécaniques.

On a donc sélectionné, dans la collection des matériaux étudiés, des couples d'échantillons, issus d'un même site, de constitutions granulométriques aussi voisines que possible mais dont la différence de teneurs en matières organiques est maximale du fait de bilans organiques très différenciés (sols de serre (3-4); Labour traditionnel - Semis direct (12-13); Prairie : surface profondeur (14-15)).

\section{RÉSULTATS}

\section{A. A l'état sec}

\section{Influence de la teneur en argile}

Les 17 échantillons présentés dans le tableau 1 permettent de mettre en évidence la relation qui existe entre la résistance en traction moyenne, mesurée à l'état sec, et la teneur en argile (fig. 3). Ainsi la teneur en argile explique à elle seule environ 94 p. 100 de la variance, la contribution de la teneur en matières organiques restant faible dans le cas de cette collection de matériaux (tabl. 2).

Cependant la teneur pondérale en argile n'est pas, à proprement parler, une variable permettant d'expliciter de façon déterministe le comportement en traction. Une approche prenant en compte l'assemblage des particules élémentaires et la cimentation due à l'argile pourrait permettre une modélisation plus analytique du phénomène.

L'indépendance de la rupture en traction vis-à-vis du frottement entre particules, explique en partie que les différentes fractions du squelette (sables et limons) ne semblent pas influer sur la résistance en traction, lais-
$R$. en traction en $100 \mathrm{kPa}$

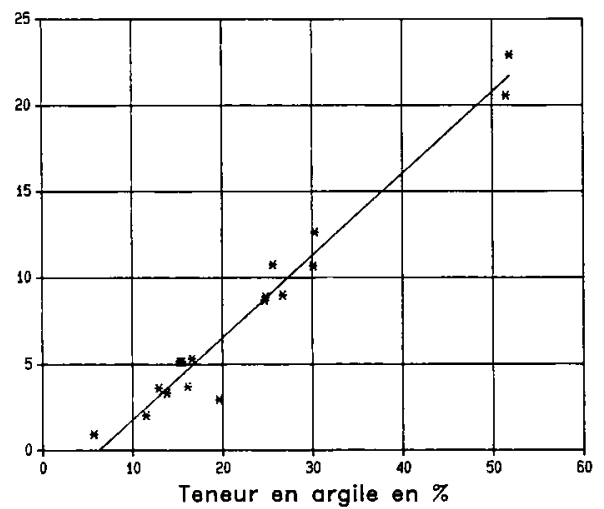

Figure 3

Relation entre la résistance en traction moyenne et la teneur en argile des échantillons de sol.

Relationship between mean tensile strength and clay content of soil sanples.

TABLEAU 2

Analyse statistique de la relation entre la résistance en traction et les teneurs en argile et en matières organiques des échantillons étudiés.

Relationship between tensile strength and the clay and organic matter contents: statistical analysis.

\begin{tabular}{|c|c|c|c|}
\hline \multicolumn{4}{|c|}{ Coefficients de corrélation - 17 échantillons } \\
\hline Variables & Argile & $\begin{array}{c}\text { Matières } \\
\text { organiques }\end{array}$ & $\begin{array}{l}\text { Résistance } \\
\text { en traction }\end{array}$ \\
\hline Argile $(\%)$ & 1,000 & & \\
\hline Mat. org. (\%) & 0,371 & 1,000 & \\
\hline Traction $\left(10^{2} \mathrm{kPa}\right)$ & 0.969 & 0.302 & 1,000 \\
\hline \multicolumn{4}{|c|}{ Paramètres des régressions } \\
\hline & \multicolumn{2}{|c|}{ Paramètres } & $\begin{array}{l}\text { Erreur } \\
\text { standard }\end{array}$ \\
\hline Argile $(\%)$ & \multicolumn{2}{|c|}{0,4752} & 0,0344 \\
\hline Mat. org. (\%o) & \multicolumn{2}{|c|}{0,0334} & 0,0344 \\
\hline Ordonnée à l'origine & \multicolumn{2}{|c|}{2,4063} & 0,9460 \\
\hline Argile (\%) & \multicolumn{2}{|c|}{0.4634} & 0,0304 \\
\hline Ordonnće à l'origine & \multicolumn{2}{|c|}{2,8947} & 0,7993 \\
\hline
\end{tabular}

sant, à la teneur en argile, un rôle prépondérant. On peut donc faire l'hypothèse que les plans de rupture se situent essentiellement dans la phase argileuse, et non à l'interface entre la phase argileuse et le squelette. La résistance en traction texturale dépendrait de la résistance en traction propre à la phase argileuse, mais aussi de l'importance des points de faiblesse que sont les lacunes.

A cet égard la teneur en argile et la taille des particules du squelette déterminent, conjointement, l'épaisseur des ponts d'argile qui cimentent l'assemblage, et sont donc responsables de la résistance en traction. Ainsi, pour les faibles teneurs en argile, inférieures à 6 p. 100 la résistance en traction reste voisine de 0 . A ces faibles teneurs en argile, on peut penser que l'hétérogénéité de la localisation des ponts d'argile entre grains et de leurs épaisseurs, peut conduire à des zones de moindre résistance, induisant globalement au niveau de l'agrégat, une plus faible résistance en traction. L'existence d'un seuil 
est apparemment fondée ; d'un point de vue statistique, car l'ordonnée à l'origine de la droite de régression entre $T$ et la teneur en argile est significativement différente de 0 au niveau 2 p. 100 . A partir d'une teneur en argile supérieure à ce seuil, la résistance en traction devient proportionnelle à la teneur en argile. Ce seuil est à rapprocher du concept de « seuil de percolation » utilisé en mécanique des milieux hétérogènes (GUYON, 1987).

Dans le «plan de rupture d'un agrégat», on peut supposer que la «surface de rupture de la phase argileuse " est pratiquement proportionnelle au complémentaire de la porosité lacunaire. Les résultats présentés figure 4 (limités à 8 échantillons par la lourdeur des mesures), tendent à montrer qu'une telle relation est envisageable.

\section{R. en traction en $100 \mathrm{kPa}$}

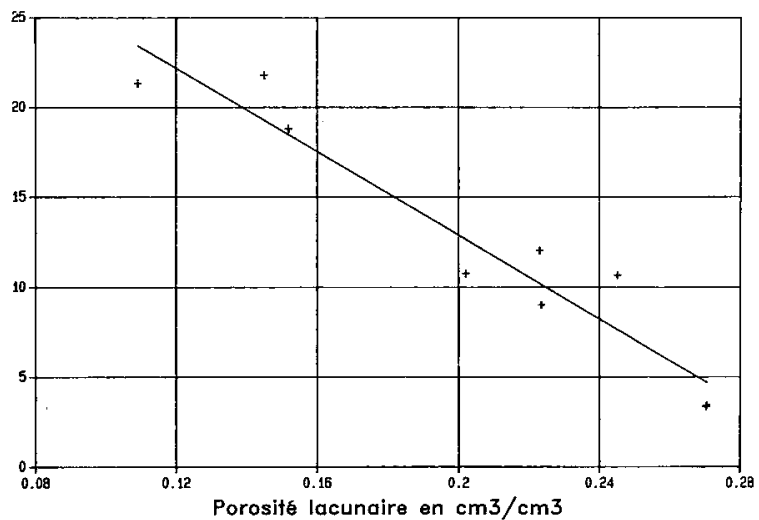

Figure 4

Relation entre la résistance en traction texturale et la porosité lacunaire déterminée par intrusion de mercure.

Relationship between textural tensile strength and lacunar porosity determined by mercury intrusion.

\section{Influence des matières organiques}

Pour mettre en évidence l'effet des matières organiques on a sélectionné, dans la population étudiée, des couples d'échantillons, prélevés dans un même site, de même granulométrie, mais qui diffèrent par leurs teneurs en matières organiques, (3-4), (12-13), et (1415), on constate ainsi (tabl. 3) de faibles différences entre les résistances en traction moyennes, mais ces différences ne sont pas également significatives.

A titre d'exemple, on compare (fig. 5) les distributions des résistances en traction (mesurées sur 280 agrégats), des échantillons $\mathrm{n}^{\circ} 12$ (Labour) et $\mathrm{n}^{\circ} 13$ (Semis-direct). On constate que l'échantillon $\mathrm{n}^{\circ} 13$, le plus riche en matiëres organiques dans le couple, présente :

- une résistance moyenne à l'écrasement, significativement plus faible,

- une distribution de la résistance en traction moins étalée: 90 p. 100 des agrégats de l'échantillon $n^{\circ} 13$ présentent une valeur de $\mathrm{T}$ inférieure à $13,6 \cdot 10^{2} \mathrm{kPa}$, alors que seulement 50 p. 100 des agrégats de l'échantillon $\mathrm{n}^{\circ} 12$ présentent une valeur de $\mathrm{T}$ inférieure à $13,5 \cdot 10^{2} \mathrm{kPa}$.

Il convient de s'assurer que cette modification des résistances en traction, est due aux différences de teneurs en matières organiques induites par les différentes

\section{TABLEAU 3}

Effet des matières organiques sur la résistance en traction movenne $T$ de matériaux de même constitution minérale. $N$ : nombre d'agrégats soumis au test.

Influence of organic matter on the mean tensile strength $T$, of soil having the same mineral constitution. $N$ : number of measurements.

\begin{tabular}{|c|c|c|c|c|c|}
\hline $\begin{array}{l}\text { Echantillon } \\
\text { (numéro) }\end{array}$ & MO & $\mathrm{MO} / \mathrm{A}$ & $\mathrm{N}$ & $\begin{array}{c}\mathrm{T} \text { moyen } \\
\mathrm{en} \\
10^{2} \mathrm{kPa}\end{array}$ & $\begin{array}{c}\text { Test } \mathrm{t} \\
\text { sur la } \\
\text { différence }\end{array}$ \\
\hline 3 & - & 0,82 & 80 & 3,47 & 0,6 \\
\hline 4 & + & 2,74 & 80 & 3,35 & non significatif \\
\hline 12 & - & 0,64 & 60 & 10,76 & 3,19 \\
\hline 13 & + & 1,44 & 60 & 9,01 & $\begin{array}{l}\text { haut. significatif } \\
\text { à } 0,2 \%\end{array}$ \\
\hline 14 & & 0,53 & 60 & 12,02 & 2,48 \\
\hline 15 & + & 0,79 & 60 & 10,64 & $\begin{array}{c}\text { significatif } \\
\text { à } 0,6 \%\end{array}$ \\
\hline
\end{tabular}

Fréquence (nombre d'agrégats)

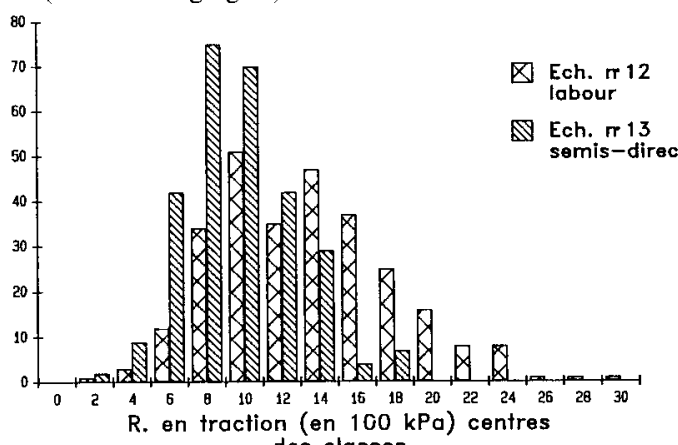

Figure 5 des classes

Comparaison des distributions des résistances en traction d'agrégats provenant de deux échantillons se différenciant essentiellement par leur teneur en matières organiques.

Comparison of the distributions of the tensile strengths of aggregates from two soil samples differing mainly in organic matter content.

techniques culturales (semis-direct/labour à $25 \mathrm{~cm}$ ), et non pas, exclusivement, à des différences de comportements physiques induites par les structures différenciées des couches de surface. Pour vérifier cette hypothèse on a procédé comme suit :

- 9 classes de résistance en traction ont été définies $a$ priori.

- Après la mesure de $\mathrm{T}$, les agrégats de même classe de résistance ont été regroupés par lots.

- Il a été effectué autant de mesures que nécessaire, pour obtenir $0,6 \mathrm{~g}$ d'agrégats par lot, masse nécessaire pour effectuer une mesure de carbone, par la méthode d'Anne (ANNE, 1945), sur chaque lot correspondant à une classe de résistance en traction.

On constate (fig. 6) qu'il existe une relation entre les résistances à l'écrasement des lots d'agrégats de l'échantillon $n^{\circ} 13$, et leurs teneurs en carbone. Par contre la variabilité des résistances en traction de l'échantillon $\mathrm{n}^{\circ} 12$ ne peut s'expliquer par celle des teneurs en carbone des lots. 
Teneur en Carbone en $\%$

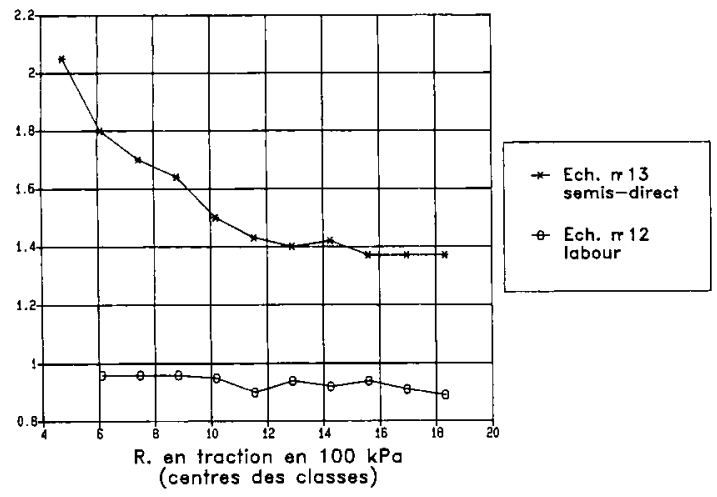

Figure 6

Mise en évidence de l'influence de la toneur en matieres organique: des agrégats sur leur résistance en traction, à partir de dosages du carbone effectués sur des lots d'agrégats de mèmes résistances en traction.

Influence of organic matler content of aggregates on their tensile strength. Carbon content measurem'nts were made on groups of aggregates having the same tensile strengths.

\section{B. A l'état humide}

Comme on l'a vu (fig. 2) le comportement des agrégats à l'écrasement peut être, en fonction de la teneur en eau, soit fragile, soit ductile. Il existe donc une gamme de teneurs en eau pour laquelle la méthode est valide, et permet d'estimer une résistance en traction. La borne supérieure de cette gamme de teneurs en eau est difficile à définir, car il s'agit d'une zone de changement progressif de comportement ne présentant, a priori, pas de discontinuité. Au-delà de cette zone de transition, on observe un fluage, et non une rupture de type «fragile".

Quand la teneur en eau augmente, la résistance en traction moyenne décroît très rapidement puis tend asymptotiquement vers 0 . De manière à pouvoir comparer les différents échantillons, on a exprimé la teneur en eau par rapport à leur teneur en argile (fig. 7). Le rapport W/A représente la teneur en eau de la phase argileuse, on peut en effet, pour cette gamme de teneur en eau, admettre que toute l'eau présente dans l'échantillon est contenue dans la phase argileuse.

R. en traction en $100 \mathrm{kPa}$

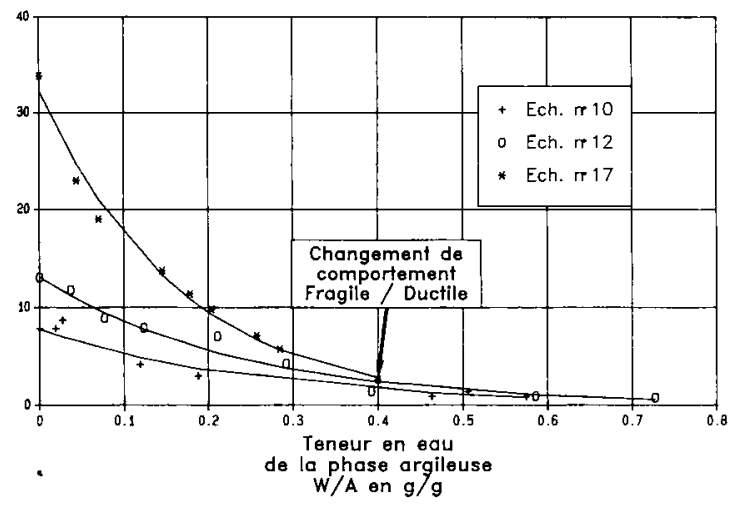

Figure 7

Variation de la résistance en traction avec la teneur en eau de la phase argileuse, pour différentes textures.

Variation with water comtent of the tensile strength of soils with different textures.
Le passage de l'état fragile à l'état ductile, estimé à partir de l'allure de l'enregistrement des variations, en fonction du temps, de la force appliquée aux agrégats, apparaît pour une gamme de teneur en eau de la phase argileuse donnée, dans les conditions de l'essai. Pour les échantillons étudiés, et dans nos conditions de mesure, on constate ainsi (fig. 7) que cette zone de transition se situe pour des teneurs en eau correspondant à une teneur en eau moyenne de la phase argileuse comprise entre 0,3 et $0,4 \mathrm{~g} / \mathrm{g}$, et un potentiel hydrique des échantillons compris entre $5 \cdot 10^{2}$ et $10 \cdot 10^{2} \mathrm{kPa}$.

Par ailleurs, les mesures étant bornées par l'humidité définie par l'équilibre de potentiel imposé par le silicagel, on considèrera, par la suite, l'humidité lors de la mesure corrigée de l'humidité après dessiccation sous silicagel: $\left(\mathrm{W}-\mathrm{W}_{0}\right) / \mathrm{A}$.

Un ajustement exponentiel décrit de façon satisfaisante la relation entre la résistance en traction et la teneur en eau (tabl. 4) :

$$
\mathrm{T}_{\mathrm{W}}=\mathrm{T}_{0}^{\prime} \cdot \mathrm{e}^{\mathrm{k}\left[\left(\mathrm{W}-\mathrm{W}_{0}\right) / \mathrm{A}\right]}
$$

où :

- $\mathrm{T}_{\mathrm{W}}$ est la résistance en traction à une humidité $\mathrm{W}$,

- $\mathrm{W}_{0}$ est l'humidité après dessiccation sous silicagel,

- A est la teneur en argile,

- $\mathrm{T}^{\prime}{ }_{0}$, ordonnée à l'origine, est une valeur ajustée de la résistance en traction après dessiccation sous silicagel; on peut l'estimer à partir des caractéristiques de l'échantillon. Elle est donnée par la relation statistique proposée tableau 2.

\section{TABLEAU 4}

Paramètres de l'équation (4): relation entre la résistance en traction texturale el la teneur en eau du materiau.

Parameters of equation (4): relationship between textural tensile strength and soil water content.

\begin{tabular}{ccrrrr}
\hline \hline Ech. $n^{\circ}$ & $\begin{array}{c}\text { Teneur } \\
\text { en } \\
\text { argile }\end{array}$ & $\begin{array}{c}\mathrm{T}_{0} \\
\text { mesuré }\end{array}$ & $\begin{array}{c}\hat{\mathbf{T}}_{0} \\
\text { estimé }\end{array}$ & $\begin{array}{c}\mathrm{T}^{\prime}{ }_{0} \\
\text { ajusté }\end{array}$ & $\mathrm{k}$ \\
\hline 10 & 24,7 & 8,89 & 8,55 & 7,22 & 3,95 \\
12 & 25,6 & 10,79 & 8,96 & 10,76 & 4,17 \\
17 & 51,9 & 22,92 & 21,15 & 23,33 & 5,50 \\
\hline
\end{tabular}

Il vient alors :

$$
\mathrm{T}_{\mathrm{W}}=(\mathrm{cA}+\mathrm{d}) \cdot \mathrm{e}^{-\mathrm{k}\left[\left(\mathrm{W} \mathrm{W}_{\mathrm{i}}\right) / \mathrm{A}\right]}
$$

Pour les trois échantillons, pris à titre d'exemple, $\left(n^{\circ s} 10-12-17\right)$ on a reporté tableau 4 les valeurs de la résistance en traction à l'état sec sous silicagel $-T_{0}$ : mesurées, $\hat{\mathrm{T}}_{0}$ : estimées à partir de la teneur en argile, ou $\mathrm{T}_{0}^{\prime}$ : ajustées à partir de l'équation (4), ainsi que les valeurs du coefficient $k$.

- Pour les trois échantillons étudiés, le coefficient $\mathrm{k}$ varie de 3,95 à 5,5 , en fonction de la teneur en argile. Cette variation est relativement faible. On a porté (fig. 8), pour les échantillons $n^{\circ} 12$ et $n^{\circ} 17$, les courbes calculées, à partir de l'équation (4) et en prenant, pour les valeurs de $T_{0}$ les valeurs mesurées, et pour $k$ 2 valeurs extrêmes $(3,5$ et 5,5$)$. Une sous-estimation de $k$ 
Résistance en traction en $100 \mathrm{kPa}$

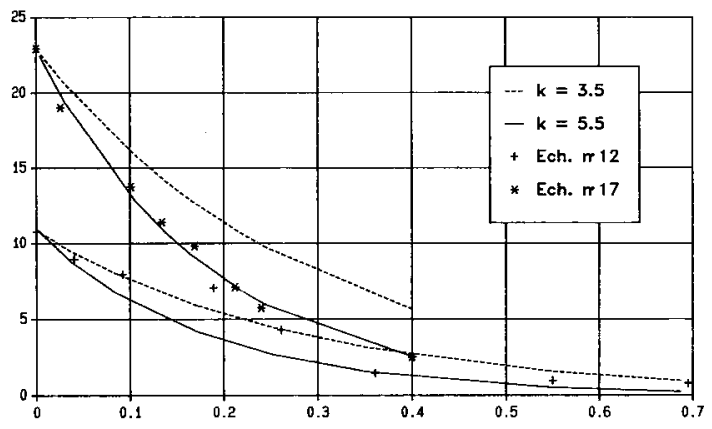

$(W-$ Wsilicagel $) / A$

Figure 8

Influence des variations du coefficient $k$ sur l'estimation de la résistance en traction en fonction de l'humidité, et de la teneur en argile.

Influence of $k$ variations on the estimate of tensile strength according to water content and clay content of the clay fabric.

$(k=3,5)$, pour le matériau le plus argileux, entraîne une surestimation de la résistance en traction pour les teneurs en eau élevées. L'écart à la valeur mesurée est alors comprise entre 2 et 3 écarts-type. Par contre une surestimation de $\mathrm{k}(\mathrm{k}=5,5)$ pour le matériau le moins argileux entraîne une sous-estimation qui reste inférieure à un écart-type quelle que soit la teneur en eau.

- On a porté figure 9 pour 2 matériaux $\left(\mathrm{n}^{\text {os }} 10-12\right)$, de teneurs en argile voisines, les points expérimentaux,

Résistance en traction en $100 \mathrm{kPa}$

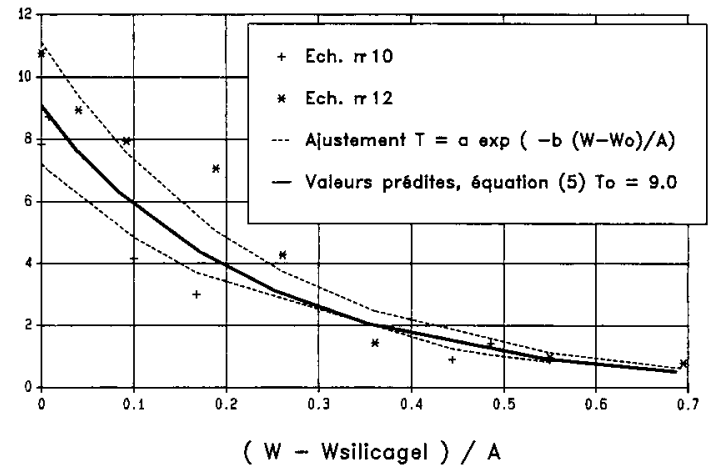

Figure 9

Influence de la qualité de l'estimation de To sur l'estimation de la résistance en traction à l'état humide.

Influence of the quality of the estimate of $T_{0}$ on the estimate of the tensile strength of wet aggregates.

les courbes ajustées par l'équation (4), et les courbes calculées par l'équation (5) à partir des teneurs en argile. L'incertitude sur $\hat{\mathrm{T}}_{0}$, estimation de $\mathrm{T}_{0}$ à partir de la seule teneur en argile, entraîne une erreur sur l'estimation de $\mathrm{T}_{\mathrm{W}}$ qui reste inférieure à l'écart-type de la mesure, quelle que soit l'humidité. Plusieurs facteurs sont susceptibles d'être à l'origine de cette incertitude sur $\hat{\mathrm{T}}_{0}$. On peut évoquer d'une part l'absence de prise en compte des caractéristiques de constitution comme la minéralogie de l'argile, et sa charge ionique, ou la quantité et la nature des matières organiques. On doit envisager, d'autre part, la microstructure des agrégats résultant de leur histoire plus ou moins récente, comme une microfissuration à l'humectation, ou le compactage de terre fine dans diverses conditions d'humidité (MONNIER \& GUERIF, 1988).

\section{DISCUSSION ET CONCLUSION}

La littérature présente peu de résultats permettant de relier la résistance en traction à la constitution des matériaux. Le génie civil, pour sa part, ne s'intéresse pas ou peu aux matériaux terreux à différents états d'hydratation et singulièrement à l'état sec. Dans le domaine de la Science du sol à applications agronomiques, si l'intérêt de l'appréciation de la résistance mécanique des sols est souvent démontré, on ne trouve pas de travaux consacrés spécifiquement à la relation résistance en traction - constitution du matériau. Ceci tient en partie au fait que les échelles auxquelles se situent les mesures rendent difficile une telle analyse.

La confrontation de nos résultats avec ceux de Williams et al., (1967) et de RogowsKi et al. (1968), Rogowski \& Don Kirkham (1976) présente un double intérêt. Celui de confirmer nos estimations, et par là même la validité de la méthode, mais aussi de montrer l'intérêt et l'originalité de la démarche.

WILliams et al. ont utilisé le test brésilien pour étudier l'effet du poly-vinyl-alcool sur la résistance en traction d'un matériau naturel remanié. Leur résultat (fig. 10) apparaît peu éloigné de l'estimation qui serait obtenue par extrapolation de la régression liant nos valeurs de résistance en traction à la teneur en argile (tabl. 2).

En cherchant à caractériser, du point de vue de leurs résistances mécaniques, les éléments structuraux présents à la surface du sol, RoGowski \& DON KIRKHAM (1976) ont évalué, la résistance en traction d'agrégats de dimensions variables.

Les résultats de RoGowsKI et al. (1968) sont présentés figure 10, les écarts constatés proviennent de ce que les mesures sont effectuées sur des agrégats, secs «à l'air », non arrondis, et de diamètres compris entre 2 et $8 \mathrm{~mm}$. Une estimation grossière des ordres de grandeur respectifs des effets de la teneur en eau et de la forme des agrégats, montre qu'ils expliquent en grande partie les différences observées. Ces remarques restent vraies visà-vis des résultats de Rogowski \& Don Kirkham (1976), présentés figure 11, qui montrent l'intérêt de définir le volume sur lequel porte la mesure de la résistance en traction. C'est le matériau le plus argileux

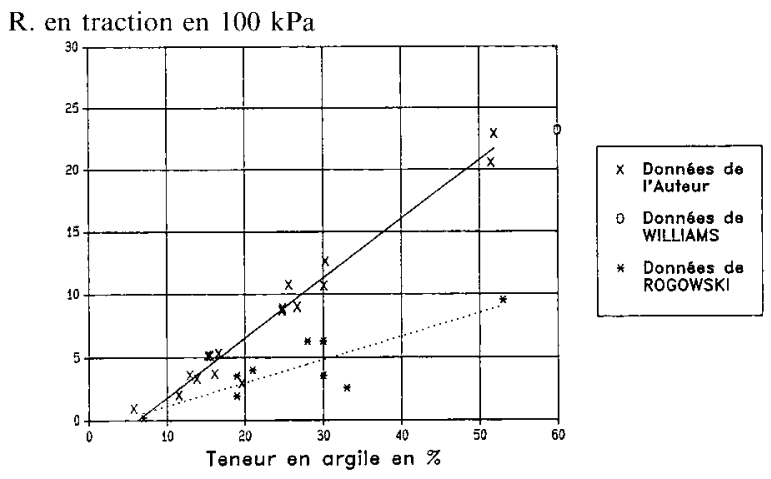

Figure 10

Comparaison des données obtenues, avec les données de la litterature. Comparison of the data obtained with data of the literature. 
Résistance en traction en $100 \mathrm{kPa}$

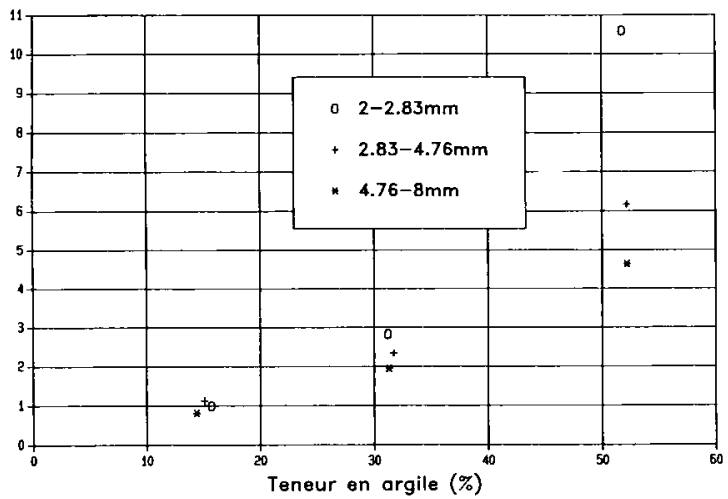

Figure 11

Influence de la taille des agrégats sur leur résistance en traction, d'après ROGOWSKI.

Influence of aggregate size on tensile strength, according to ROGOWSKI.

qui illustre le mieux l'intérêt de prendre en compte le plus petit volume représentatif possible, pour s'affranchir des effets de la micro-fissuration, et étudier la "cohésion » de l'assemblage des constituants du sol.

La qualité de la relation statistique entre la teneur en argile et la résistance en traction à l'état sec, définie à l'échelle de l'organisation texturale, présente donc un intérêt prédictif certain. Celui-ci reste limité néanmoins à des matériaux dont la constitution minéralogique, et la charge ionique, entre autres, restent voisines de celles de la population de matériaux étudiés.

L'extension de cette relation au domaine humide permet d'envisager une prévision, au moins grossière, de la résistance en traction texturale dans la gamme hydrique de validité du test.

Pour améliorer l'estimation de $\mathrm{T}_{0}$, une modélisation, à partir de la porosité lacunaire, et la résistance en traction propre à la phase argileuse, semble pouvoir se substituer à la relation statistique résistance en traction - teneur en argile.

Ce travail ouvre la possibilité d'analyser la résistance en traction de milieux fragmentaires et agrégés, en combinant des évaluations de résistances en traction à différentes échelles.

Reçu le 19 novembre 1987. Accepté le $1 /$ mars 1988.

\section{REMERCIEMENTS}

Ce travail a été en grande partie effectué dans le cadre de l'A.T.P. tripartic C.N.R.S. - I.N.R.A. - M.E.R./M.E.C.V. : MATIÈRES ORGANIQUES DANS LES SOLS, dont la coordination était assuréc par le PIREN/C.N.R.S. (1984-1986).

\section{RÉFÉRENCES BIBLIOGRAPHIQUES}

Anne P., 1945. Dosage du carbone organique du sol. Ann. agron. Sér. A, p. 165.

Braunack M. V., Hewitt J. S., Dexter A. R., 1979. Brittle failure of soil aggregates and the compaction of aggregate beds. J. Soil Sci., 30, 653-668.

Dexter A. R., 1975. Uniaxial compression of ideal brittle tilths. $J$. Terramechanics, 12 (1), 3-14.

Farrel D. A., Greacen E. L., Larson W. E., 1967. The effect of water content on axial strain in a loam soil under tension and compression. Soil Sci. Am. Proc., 31, 445-450.

Fiès J. C., Stengel P., 1981. Densité texturale des sols naturels. I. Méthode de mesure. Agronomie, 1 (8), 651-658.

Fiès J. C., 1984. Analyse de la répartition du volume des pores dans les assemblages argile-squelette : comparaison entre un modèle d"espace poral textural et les données fournies par la porosimétrie au mercure. Agronomie, 4 (9), 891-899.

Frydman S., 1964. The applicability of the Brazilian (indirect tension) test to soils. Aust J. appl. Sci., 15, 335-343.

Godwin R. J., Spoor G., 1977. Soil failure with narrow tines. J. agric : Eng. Res., 22, 213-228.

Guérif J., 1988. Détermination de la résistance en traction des agrégats terreux : revue bibliographique et mise au point technique. Agronomie, 8 (4), 281-288
Guyon E., 1987. La matière en grand désordre. In J. L. Gourdon, (Ed.), Les milieux granulaires, p. 7-14. Journées Physique des Ponts et Chaussées, Les Arcs, France, 14-17 décembre 1987.

Hiramatsu Y., Oka Y., 1966. Determination of the tensile strength of rock by a compression test of an irregular test piece. Int. J. Rock Mech. Min. Sci., 3, 89-99.

Monnier G., Guerif J., 1988. Conséquences physiques et agronomiques du tassement. C.R. Ac'ad. Agric. Fr, Séance du 20 janvier 1988 (à paraître).

Quikk J. P., Panabokke C. R., 1962. Incipient failure of soil aggregates. J. Soil. Sci., 13, 60-69.

Quirk J. P., Panabokke R., 1962. Incipient failure of soil aggregates. J. Soil Sci., 13 (1), 60-70.

Rogowski A. S., Moldenhauer W. C., Don Kirkham, 1968. Rupture parameters in soil aggregates. Soil Sci. Soc ; Am. Proc., 32, 720-724.

Rogowski A. S., Don Kirkham, 1976. Strength of soil aggregates : influence of size, density and clay and organic matter content. Med. Fac. Landbouww. Rijksuniv. Gent, 41 (1), 85-100.

Sternberg E., Rosenthal F., 1952. The elastic sphere under concentrated loads. J. appl. Mech., 19, 413-421.

Williams B. G., Greenland D. J., Quirk J.P., 1967. The tensile strength of soil cores containing polyvinyl alcohol. Aust. I. Soil Res., $5,85-92$ 